\title{
Ludwigsburger Beiträge zur Medienpädagogik
}

Online-Magazin des Interdisziplinären Zentrums für

Medienpädagogik und Medienforschung an der PH Ludwigsburg

\section{Cyber-Grooming \\ Präventionskonzepte zum Schutz von Grundschüler*innen}

\section{Jennifer Vogel}

Dieser Beitrag stellt die aktualisierte und gekürzte Fassung der gleichnamigen Bachelorarbeit der Autorin dar, die im Rahmen des Bachelorstudiengangs Lehramt für Grundschulen an der Pädagogischen Hochschule in Ludwigsbug verfasst wurde.

\section{Zusammenfassung des Beitrags}

Der vorliegende Beitrag ermöglicht Einblicke in bislang in geringem Maße erforschte Handlungsstrategien von Grundschüler*innen, wenn diese mit Cyber-Grooming konfrontiert werden. Um sich der Forschungslücke zu nähern, wurden sechs Viertklässler*innen mithilfe eines qualitativen Leitfadeninterviews und eines ergänzenden Kurzfragebogens zur eigenen Mediennutzung und ihren Verhaltensweisen im Internet befragt. Dabei konnte eine intensive Auseinandersetzung mit digitalen Medien festgestellt werden. Explizites Wissen zu Cyber-Grooming war bei den Grundschüler*innen jedoch nicht vorhanden. Ferner wurde eine starke Variation in Bezug auf Verhaltensweisen bei risikoreicher Online-Kommunikation sichtbar. Insgesamt wird deutlich, dass sowohl hinsichtlich der intensiven Nutzung des Internets als auch aufgrund unsicherer Handlungsstrategien in Online-Kommunikationssettings eine umfassende Medienbildung in schulischen Kontexten unerlässlich ist.

Schlïsselbegriffe: Medienpädagogik • Cyber-Grooming • Prävention $\bullet$ Medienforschung

\section{Einführung in die Thematik}

„ich weiß mit 100-prozentiger sicherheit, dass du bestimmt schon an dir rumgespielt hast... das ist doch die natur... du siehst oder börst etwas, was dich geil macht, du wirst feucht und würdest es dir am liebsten machen" (Enders 2004, S. 7)

Manch Unwissender würde der Tatsache, dass die angeführte Äußerung in einem Chatroom für Kinder gefallen ist, wohl keinen Glauben schenken. Die hauptamtlichen Mitarbeiter des Vereins Zartbitter recherchierten im Jahre 2004 verdeckt in speziell für Minderjährige vorgesehenen Chaträumen (vgl. Enders 2004, S. 1). Hierbei konnten sie feststellen, dass sexuell geprägte Aussagen in Online-Kommunikationssettings scheinbar keine Seltenheit sind (vgl. Enders 2004, S. 3). ${ }^{1}$ Link für weitere Informationen zu den Arbeitsschwerpunkten von Zartbitter: https://www.zartbitter.de/gegen_se-
xuellen_missbrauch/Aktuell/Wir_ueber_uns.php

J. Vogel. Cyber-Grooming. Präventionskonzepte zum Schutz von Grundschüler*innen. Ausgabe 20/2019 
Heranwachsende leben in einer Welt, in der digitale Medien und die damit verbundenen Nutzungsmöglichkeiten den Alltag maßgeblich beeinflussen (vgl. Roll 2017, S. 13). Neben klassischen Chaträumen ist Kommunikation und Austausch auch durch die Vernetzung über Soziale Netzwerke möglich (vgl. Roll 2017, S. 14). Bereits Grundschüler*innen scheint es leicht zu fallen, mithilfe von digitalen Medien Freundschaften aufrechtzuerhalten oder neue Kontakte zu knüpfen.

Bei Betrachtung der zu Beginn angeführten Aussage wird deutlich, dass nicht alle Kommunikationspartner im Internet harmlose Alltagsgespräche anstreben. Welchen Gefahren und Risiken Kinder im Bereich der Online-Kommunikation ausgesetzt sein können, darf aus diesem Grund nicht bagatellisiert werden (vgl. Roll 2017, S. 6). Neben zufällig wirkenden und isoliert auftretenden sexuellen Belästigungsformen existiert eine weitaus manipulativere Weise, die Täter anwenden, um sexuelle Bedürfnisse auf Kosten von Minderjährigen zu befriedigen.

Cyber-Grooming nennt sich das Phänomen, bei dem Erwachsene in Online-Kommunikationssettings über einen längeren Zeitraum Vertrauen aufbauen, um Kinder und Jugendliche anschließend für eigene sexuelle Absichten zu missbrauchen. Durch die wachsende Anzahl virtueller Kommunikationsmöglichkeiten wachsen auch Wege, anonym und somit ungehemmt miteinander kommunizieren zu können (vgl. Katzer 2014, S. 3). Eine intensive Nutzung digitaler Medien und des Internets von Kindern und Jugendlichen erhöht die Gefahr, mit möglichen Tätern in Kontakt zu kommen (vgl. Gottschalk 2011, S. 447).

Um passende Präventionsgrundlagen zu schaffen und potenziell Betroffenen ${ }^{2}$ Schutz vor Viktimisierung zu bieten, eignen sich schulische Institutionen. Bereits in der Grundschule können grundlegende Kompetenzen im Bereich der Medienpädagogik gefördert werden. Eine Vorgehensweise zur Konzeption zielführender Präventionsmaßnahmen kann die Überprüfung und Beurteilung von bereits vorhandenen Handlungsstrategien von Kindern in Gefahren- und Risikosituationen im Internet darstellen. Auf diese Weise können Defizite aufgedeckt und gezielt ausgeglichen werden.

Entsprechende Forschungsarbeiten zu Handlungsstrategien von Kindern sowie präventive Maßnahmen zum Schutz vor Cyber-Grooming existieren bislang lediglich vereinzelt. Insbesondere Grundschüler*innen wurden dabei kaum in den Blick genommen. Angesichts dieses Forschungsdefizits lautet die Forschungsfrage des vorliegenden Beitrags: Wie können Präventionsmaßnahmen konzipiert werden, um Grundschüler*innen auf Grundlage ibrer Handlungsstrategien vor Cyber-Grooming zu schützen? Mithilfe der gestaltungsorientierten Forschungsfrage sollen Praxismaßnahmen für Präventionskonzepte herausgearbeitet werden, die zum Schutz von Grundschüler*innen vor Cyber-Grooming beitragen können.

\section{Theoretischer Hintergrund}

\subsection{Cyber-Grooming}

Der Begriff Cyber-Grooming, auch als Online-Grooming bezeichnet (vgl. Wachs 2017, S. 152), besitzt ein weites Spektrum an unterschiedlichen Definitionsansätzen. Eine allgemeingültige Definition des Phänomens existiert jedoch nicht (vgl. Dekker/Koops/Briken 2016, S. 28).

\footnotetext{
2 In vorliegender Arbeit wird der Begriff des Opfers bewusst durch den der Betroffenen ersetzt, da auf diese Weise ein respektvoller und distanzwahrender Umgang gegenüber denjenigen, die der Bezeichnung kritisch gegenüberstehen, gewährleistet werden kann.
} 
Bei Zerlegung des Begriffes in seine Bestandteile Cyber und Grooming bezeichnet das Präfix Cyber die virtuelle Welt des Internets (auch: Cyber-Space) und somit den Ort des stattfindenden Prozesses (vgl. Freelance 2018, S. 2). Abgeleitet vom englischen to groom kann der Begriff Grooming anschließend sinngemäß durch pflegen oder vorbereiten übersetzt werden (vgl. UBSKM 2019, o. S.). Cyber-Grooming beschreibt somit einen Anbahnungsprozess im Internet, bei dem ein spezifisches Ziel vorbereitet wird.

Nach der Definition von Webster et al. (2012) wird Cyber-Grooming wie folgt definiert:

Online-Grooming ist ein Prozess, bei dem eine Person sich mit einer jüngeren Person (in dieser Definition 16 Jahre oder jünger) online anfreundet, um Gelegenheit für sexuellen Online-Kontakt und/oder ein physisches Treffen zu schaffen, mit dem Ziel, sexuellen Missbrauch zu begehen (Webster et al. 2012, S. 5, Übers. JV).

Cyber-Grooming bezeichnet also die Vorgehensweise von Erwachsenen, in Online-Kommunikationssettings Kontakt zu Kindern und Jugendlichen aufzunehmen und deren Vertrauen durch gezielte Manipulation zu erschleichen. Dabei steht die Vorbereitung des sexuellen Missbrauchs im Vordergrund (vgl. Wachs 2017, S. 50; UBSKM 2019, o. S.).

Anzumerken ist an dieser Stelle, dass Cyber-Grooming kein neues Phänomen der digitalen Medien darstellt. Das Vorbereiten einer Missbrauchstat ist auch in Fällen des Kindesmissbrauchs der Offline-Welt bekannt (vgl. Bullens 1995, S. 55). Einzelne Definitionsansätze lehnen sich daher an diese Anbahnungen an (vgl. Wachs 2017, S. 49).

\section{Täter*innenmerkmale}

Bei Cyber-Grooming-Tätern handelt es sich hauptsächlich um erwachsene Männer. Weibliche Täterinnen sind durchaus existent, allerdings ist dieser Prozentsatz gering, sodass lediglich vereinzelt Informationen vorliegen (vgl. Enders 2004, S. 11). Das Alter kann grob durch die Altersspanne zwischen 18 und 40 Jahren eingegrenzt werden, allerdings sind auch Täter*innen unter 18 Jahren bzw. über 40 Jahren bekannt (vgl. Wachs 2017, S. 168). Das Internet wird von allen sozialen Schichten genutzt. Diese Mischung ist auch bei Grooming-Täter*innen vertreten (vgl. Enders 2004, S. 10; Webster et al. 2012, S. 6). Auf eine bestimmte Personengruppe kann daher prinzipiell nicht geschlossen werden.

\section{Ziele, Kontaktaufnabme und Grenæüberschreitung}

Die Ziele der Täter*innen liegen in den meisten Fällen bei persönlichen Treffen, bei denen der sexuelle Missbrauch im Vordergrund steht (vgl. Wachs 2017, S. 50). Jedoch kann auch der reine Austausch von Nachrichten oder Bild- und Videomaterial Ziel des CyberGroomings sein (vgl. Katzer 2014, S. 2; UBSKM 2019).

Der Kontakt wird über Chaträume und Soziale Netzwerke hergestellt (vgl. Wachs 2017, S. 52). Wenn durch Kommunikation eine Vertrauensbasis erreicht werden konnte, nutzen Täter*innen diese für ihre eigentlichen Absichten (vgl. Freelance 2018, S. 4). In dieser Phase folgt die Grenzüberschreitung der Gefühle der Betroffenen. Die Grooming-Täter*innen konfrontieren die Kinder mit sexuell geprägtem Bild- oder Nachrichtenmaterial oder verlangen eigene Aufnahmen der Betroffenen (vgl. Wachs 2017, S. 53). Durch Manipulation und Erpressung kann es den Täter*innen gelingen, dass die Betroffenen dem nachgeben. In manchen Fällen wird schließlich versucht, ein persönliches Treffen zu vereinbaren (vgl. Freelance 2018, S. 3). 


\section{Risikofaktoren von Betroffenen}

Prinzipiell ist zu sagen, dass alle Kinder, die digitale Online-Medien und Social Media-Anwendungen nutzen, von Cyber-Grooming betroffen sein können. Anders als bei sexuellem Missbrauch in der Offline-Welt, bei dem die Risikogruppe aufgrund von Biografie, Geschlecht oder Bildungsgrad näher eingegrenzt werden kann, können diese Faktoren in der Online-Welt bedeutungslos sein (vgl. Katzer 2007, S. 81).

\subsection{Prävention}

Der Begriff Prävention bezeichnet Maßnahmen, die zum Schutz vor Gefahren und Risiken beitragen können und somit physische und psychische Gesundheit potenziell Gefährdeter sicherstellen sollen (vgl. Wachs 2017, S. 172). Grundsätzlich konzentrieren sich Ansätze moderner Präventionskonzepte auf „Aufklärung, Information und Empowerment von Risikogruppen“ (Wachs 2017, S. 172). Reine Abschreckung sowie Präventionsmaßnahmen, die ausschließlich auf Verboten basieren, sind nicht zielführend und sollten daher vermieden werden (vgl. Wachs 2017, S. 172; UBSKM 2019). Um eine kompetente Nutzung digitaler Medien zu ermöglichen, muss Präventionsarbeit fokussiert werden, bei der Aspekte von „Anregung, Unterstützung, Ermutigung [und] Stärkung der eigenen Kräfte“ (Braun 2001, S. 123) im Vordergrund stehen. Ein vollständiger Schutz vor Betroffenheit kann durch Präventionsmaßnahmen allerdings nicht garantiert werden (vgl. Buskotte 2001, S. 60).

\section{Schülerbefragung}

\subsection{State of the Art: Handlungsstrategien bei Cyber-Grooming}

Handlungsstrategien von Grundschüler*innen in Bezug auf Cyber-Grooming wurden in der Vergangenheit lediglich vereinzelt untersucht (vgl. Vogelsang 2017, S. 319). Anhand vorhandener Forschung zu anlehnenden Themenbereichen ist es jedoch möglich, Rückschlüsse zu ziehen.

Mit dem Ziel, Handlungsstrategien bei Konfrontation mit Cyber-Grooming aufzudecken, befragten Wachs, Wolf und Pan (2012) im Jahre 2011 insgesamt 518 Fünft- bis Zehntklässler*innen vier unterschiedlicher Schulen (vgl. Wachs/Wolf/Pan 2012, S. 629).

Durch die Befragung konnten drei Dimensionen verschiedener Handlungsstrategien herauskristallisiert werden. Die Verhaltensweisen bei sexueller Online-Viktimisierung lassen sich anhand der angeführten Forschung in kognitiv-technisches Handeln, aggressiv-selbstbehauptendes Handeln und bilfloses Handeln gliedern (vgl. Wachs/Wolf/Pan 2012, S. 631).

Bei weiterer Recherche war festzustellen, dass erforschte Handlungsweisen anderer Forschungsergebnisse, die in keine übergeordneten Typen kategorisiert wurden, den Typen von Wachs, Wolf und Pan (2012) untergeordnet werden können. Aus diesem Grund werden die einzelnen Typen bei nachfolgender Beschreibung durch weitere Studienergebnisse ergänzt.

Die in der Studie von Wachs, Wolf und Pan (2012) sichtbar gewordenen kognitiv-technischen Handlungen charakterisieren sich durch die Beleuchtung der Intention der Täter*innen. Die Schüler*innen hinterfragen bei dieser Handlungsdimension die Absicht der Kommunikation (vgl. Wachs/Wolf/Pan 2012, S. 631). Zudem werden Handlungsweisen deutlich, bei denen versucht wird, den Kontakt zum/zur Täter*in gezielt abzubrechen, indem darum gebeten wird, die Kommunikation zu beenden. Auch spielen technologische Verhaltensweisen, wie das Herunterfahren des Computers oder die Veränderung des eigenen Accounts, eine Rolle (vgl. Wachs/Wolf/Pan 2012, S. 631). 
Grimm, Rhein und Clausen-Muradian (2008) konnten bereits vier Jahre zuvor Strategien bei Gewalterfahrungen im Internet offenlegen (vgl. Grimm/Rhein/Clausen-Muradian 2008, S. 63). Die Jugendlichen im Alter von 13 bis 21 Jahren gaben bei der Befragung an, auf sexuelle Online-Belästigung mit Ignoranz zu reagieren oder die Blockier-Funktion zu nutzen (vgl. Grimm/Rhein/Clausen-Muradian 2008, S. 255). Insbesondere das Nutzen der Blockier-Funktion lässt sich als technische Verhaltensweise in die kognitiv-technische Handlungsdimension von Wachs, Wolf und Pan (2012) eingliedern.

Zusätzlich lassen sich Ergebnisse der Forschung von Vogelsang (2017) in diese Rubrik einordnen. Die Studie untersuchte Handlungsstrategien bei sexueller Online-Viktimisierung von insgesamt 302 Jugendlichen im Alter von 14 bis 17 Jahren. Bei der Online-Befragung mit Möglichkeiten der Mehrfachnennung gaben über 70 Prozent der Jugendlichen an, entsprechende Kommunikationspartner bei sexueller Belästigung im Internet zu blockieren (vgl. Vogelsang 2017, S. 220). 60 Prozent der Befragten nannten zusätzlich das Verlassen des Chats als Handlungsoption (vgl. Vogelsang 2017, S. 221). Die Mehrheit der befragten Jugendlichen wendet somit technologische Handlungen an, um die Kommunikation zu beenden.

Eine weitere Handlungsdimension stellt das aggressiv-selbstbehauptende Verhalten dar (vgl. Wachs/Wolf/Pan 2012, S. 631). Hierunter fallen Handlungen wie die deutliche Mitteilung, dass die Kommunikation nicht erwünscht ist. Auch werden Beleidigungen und Drohungen ausgesprochen (vgl. Wachs/Wolf/Pan 2012, S. 631). Grimm, Rhein und Clausen-Muradian konnten in ihrer Studie ebenfalls Strategien erforschen, die das Beleidigen des Kommunikationspartners aufweisen (vgl. Grimm/Rhein/Clausen-Muradian 2008, S. 255). Bei Vogelsang war eine Auswahloption, die Beleidigungen und aggressives Verhalten beinhaltet, nicht gegeben. 46 Prozent der befragten Jugendlichen gaben jedoch an, deutlich mitzuteilen, wenn keine weitere Kommunikation gewünscht ist (vgl. Vogelsang 2017, S. 221).

Der von Wachs, Wolf und Pan erforschten hilflosen Dimension können Strategien zugeordnet werden, die unangenehme Online-Situationen in geringem Maße zielführend lösen. Hierbei wurden an die Täter*innen gerichtete, verzweifelte Bitten ausgesprochen, um die Kommunikation zu beenden, oder resultierend aus Unwissenheit keine Strategien angewandt (vgl. Wachs/Wolf/Pan 2012, S. 631). Auch in der Studie von Vogelsang gaben 5 Prozent der Jugendlichen an, keine weiteren Schritte einzuleiten (vgl. Vogelsang 2017, S. 219). Ob an dieser Stelle nicht gehandelt wird, da keine Notwendigkeit gesehen wird oder ob andere Gründe ausschlaggebend sind, wird nicht näher erfasst. Der Anteil dieser Jugendlichen ist insbesondere bei den jüngeren Teilnehmer*innen der Befragung hoch (vgl. Vogelsang 2017, S. 220).

Insgesamt konnte im Bereich der sexuellen Online-Belästigung festgestellt werden, dass diese Erfahrungen überwiegend als unangenehm eingestuft werden (vgl. Grimm/Rhein/ClausenMuradian 2008, S. 254). Daher werden mehrheitlich Handlungsstrategien angewandt, die das Ziel besitzen, die Kommunikation zu beenden. Erwähnenswert ist außerdem, dass knapp 10 Prozent der Jugendlichen angaben, keine Kenntnisse über mögliche Präventions- und Schutzstrategien vor sexueller Viktimisierung zu besitzen (vgl. Vogelsang 2017, S. 224). Die Relevanz der Präventionsarbeit wird durch die vorliegenden Ergebnisse verdeutlicht. Bei Betrachtung der vorliegenden Forschungsergebnisse muss beachtet werden, dass die Befragungen hauptsächlich Schüler*innen der Klassenstufen 5 bis 12 fokussierten. Die Ergebnisse können somit aufgrund des Altersunterschieds der Befragten für Grundschüler*innen 
keine Repräsentativität gewährleisten. Im Idealfall kann aufgrund der Alters- und Klassenstufe davon ausgegangen werden, dass die befragten Schüler*innen bereits mit Medienkompetenzförderung und Präventionsarbeit in Kontakt gekommen sind (beispielsweise in der weiterführenden Schule). An dieser Stelle wäre zu erwarten gewesen, dass die Schüler*innen einen kompetenten Umgang mit digitalen Medien aufweisen. Es wird deutlich, dass Präventionsmaßnahmen, die bereits in der Grundschule Grundlagen schaffen können, relevant sind, um Schüler*innen Möglichkeiten bezüglich zielführender Schutz- und Handlungsstrategien zu vermitteln.

\subsection{Zielsetzung}

Die Überschaubarkeit des aktuellen Forschungsstands zu Handlungsstrategien von Grundschüler*innen bei Konfrontation mit Cyber-Grooming verdeutlicht, dass weitere Forschungsarbeiten notwendig sind, insbesondere um auf dieser Grundlage (weitere) Präventionskonzepte zu entwickeln.

Mithilfe einer eigenen Studie wurde im Rahmen einer qualitativen Befragung erforscht, welche Handlungsstrategien Grundschüler*innen bei risikoreicher Online-Kommunikation schildern. Auf Grundlage dieser Schilderungen sollten weiterführende Überlegungen ermöglicht werden, auf welche Weise Präventionsmaßnahmen zum Schutz vor Cyber-Grooming konzipiert werden können. Zielsetzend sollten diese Maßnahmen die von den Grundschüler*innen genannten Handlungsstrategien aufgreifen und optimieren, um somit abgestimmt auf individuelle Bedürfnisse bestmöglichen Schutz vor Cyber-Grooming zu gewährleisten.

\subsection{Methodik}

Um Einblicke in die Forschungsfrage zu erhalten, wurden Leitfadeninterviews mit mehreren Grundschüler*innen geführt. Ein begleitender Kurzfragebogen ermöglichte neben biografischen Daten zusätzlich Einsichten in die Medienausstattung und Mediennutzung der befragten Kinder.

Die Interviews wurden mit insgesamt sechs Schüler*innen einer Grundschule im Mai 2019 durchgeführt. Mithilfe eines integrierten Theaterspiels konnte eine Chatsituation simuliert werden, die das Thema Cyber-Grooming spielerisch kindgerecht durch Unterstützung einer Handspielpuppe einführt. Auf diese Weise wurden die Grundschüler*innen nicht mit schockierenden Inhalten konfrontiert (vgl. Gläser/Laudel 2010, S. 51).

Alle befragten Schüler*innen besuchten die vierte Klassenstufe. Neben organisatorischen Gründen beeinflussten auch Faktoren der KIM-Studie, in der das Medienkonsumverhalten mit zunehmendem Alter gesteigert wird (vgl. KIM-Studie 2018), die Festlegung auf die gewählte Klassenstufe. Die Teilnehmer*innen meldeten sich freiwillig. Aus zeitlichen Gründen wurde eine Gruppenbefragung mit jeweils zwei Schüler*innen pro Interview durchgeführt. Die subjektive Sicht jedes Kindes stand jedoch weiterhin im Fokus, sodass Dynamiken der Gruppe nicht untersucht wurden (vgl. Schreier 2004, S. 381).

\section{Datenauswertung}

Um detaillierte Einblicke in die Forschungsfrage zu erhalten, wurde das erhobene Datenmaterial ausgewertet und analysiert. Die vorliegende Studie kann keine Repräsentativität für sich in Anspruch nehmen.

Weiterhin ist zu beachten, dass die beschriebenen Handlungsstrategien keine durchgeführten oder beobachteten Verhaltensweisen darstellen. Durch die Simulation des Theaterspiels sind lediglich Was wäre, wenn ...? - Situationen gegeben. Die Grundschüler*innen gaben somit an, 
dass sie in bestimmten Fällen auf bestimmte Weise reagieren würden. Ferner noch wurden bestimmte Verhaltensweisen lediglich als Ratschlag an die Puppe Clara weitergegeben. Dennoch eignet sich die Befragung, um erste Einblicke in Handlungsstrategien von Grundschüler*innen zu erhalten.

\section{Darstellung der Ergebnisse}

\subsection{Mediennutzung der befragten Grundschüler*innen}

Mithilfe des Kurzfragebogens konnten Einblicke in die Mediennutzung der befragten Grundschüler*innen ermöglicht werden. Ein gekürzter Überblick wird in folgendem Abschnitt dargestellt.

Im Bereich der Medienausstattung ist sowohl der Computer/Laptop als auch ein Tablet laut Angaben der Grundschüler*innen in allen Haushalten vorhanden. Fünf der sechs Kinder geben an, über ein eigenes Smartphone zu verfügen. Deutlich wird, dass zahlreiche Geräte, mit denen der Zugriff auf das Internet möglich ist, bei den Kindern vorhanden sind.

Alle befragten Teilnehmer*innen geben an, zuhause über Zugang zum Internet zu verfügen. Ein Drittel darf diesen Zugriff ohne Beschränkung nutzen. Alle Smartphone-Besitzer*innen können auch mit diesem zuhause online gehen. Zwei der befragten Schüler*innen haben unterwegs Zugriff auf das Internet. Online sind alle Teilnehmer*innen laut eigener Angabe jeden Tag, mehrheitlich ohne Begleitung anderer Personen. Lediglich ein Schüler nutzt das Internet hauptsächlich gemeinsam mit Geschwistern.

Dominierend ist bei der Nutzung des Internets die Plattform YouTube. Alle befragten Schüler*innen berichten, diese jeden Tag zu verwenden. Auch der Kommunikationsmessenger Whats App wird von allen Smartphone-Besitzern jeden Tag genutzt.

Zusammenfassend kann gesagt werden, dass die Medienausstattung innerhalb der Stichprobe umfangreich ausfällt. Alle befragten Grundschüler*innen besitzen Geräte, die einen Zugriff auf das Internet ermöglichen. Es liegt eine intensive Nutzung von Online-Plattformen vor, durch die eine potenzielle Kontaktaufnahme mit Täter*innen möglich ist. Grundschüler*innen müssen daher insbesondere bei Betrachtung der überwiegend autonomen Nutzung bezüglich Verhaltensweisen im Internet gefördert und unterstützt werden. Auf Nachfrage im Verlauf des Interviews erwähnte ein Schüler, schon einmal von einer unbekannten Person angeschrieben worden zu sein, den Kontakt allerdings erfolgreich beendet zu haben.

\subsection{Handlungsstrategien der befragten Grundschüler*innen}

Im folgenden Abschnitt werden Verhaltensweisen und Handlungsstrategien in Online-Kommunikationssettings der befragten Grundschüler*innen dargestellt, die mithilfe des Theaterspiels erkenntlich gemacht werden konnten.

\section{Gefahren und Risiken bei der Online-Kommunikation}

Schüler*innen können in Online-Kommunikationssettings mit Gefahren und Risiken wie der Begegnung mit Cyber-Grooming konfrontiert werden. In der Interviewsituation trifft die Handpuppe in einem Chatraum auf ein ihr unbekanntes User-Profil, das sie zunächst nach ihrem Befinden befragt. Im Laufe der Kommunikation werden persönlichere Details sowie intime Bilder gefordert. 
Bei Betrachtung der Aussagen der befragten Grundschüler*innen wird deutlich, dass sich die Mehrheit mit den möglichen Intentionen von unbekannten Chatpartnern auseinandersetzt. Welches Ziel der Kommunikationspartner durch die Kontaktaufnahme verfolgen könnte, besonders wenn dabei kein relevanter Grund gegeben ist, wird schon bei Chatbeginn kritisch in den Blick genommen. Die Intention des Chatpartners wird von den Grundschüler*innen durchaus als betrügerisch angesehen, auch wenn noch kein ausschlaggebender Grund vorhanden ist. Obwohl der unbekannte User-Account die Handpuppe zunächst nur nach ihrem Wohlergehen fragt, stehen die Grundschüler*innen dem Chatpartner kritisch gegenüber und vermuten bösartige Absichten.

Im Setting der Theaterspielsituation schätzen die Grundschüler*innen vor allem den Austausch und anschließenden Diebstahl von eigenen persönlichen Daten als gefährlich ein. Vermutet wird, dass der Kommunikationspartner Informationen wie Telefonnummern oder Adressen ausfindig machen möchte. Bei expliziter Nachfrage nach dem eigenen Wohnort nehmen die Schüler*innen an, dass der Kommunikationspartner einen Einbruch begehen will. Auch die Gefahr eines Hackerangriffs wird bei der Nachfrage nach persönlichen Daten in Erwägung gezogen.

Hinter der Kontaktaufnahme wird auch die Intention eines Treffens in der Offline-Welt vermutet. Die Schüler*innen erkennen, dass bösartige Absichten von Kommunikationspartnern im Internet nicht immer ausschließlich die reine Online-Kommunikation betreffen, sondern auch persönliche Treffen angestrebt werden. Dabei werden vor allem mediengeprägte Vorstellungen einer Entführung - beispielsweise der Transport mit einem Van - geäußert. Eine sexuelle Intention wird bezogen auf das persönliche Treffen allerdings nicht erwähnt. Generell werden sexuelle Absichten zu keinem Zeitpunkt des Interviews angesprochen. Auch bei expliziter Aufforderung, ein in Unterwäsche bekleidetes Bild zu versenden, erwähnen die Schüler*innen nicht, dass der Kommunikationspartner durch diese Forderung sexuelle Ziele verfolgen könnte.

Ein Grundschüler deutet als mögliche Intention der Kontaktaufnahme die gezielte Suche von Erwachsenen nach jüngeren Kindern an. Er nennt somit eine spezielle Zielgruppe von Chatpartnern. Eine explizite Gefahr, die von dieser Intention ausgeht, beispielsweise CyberGrooming oder gezielte Manipulation von Minderjährigen, auch in Bezug auf sexuelle Absichten, thematisiert der Schüler allerdings nicht.

Zusammenfassend sind der Mehrheit der Grundschüler*innen potenzielle Gefahren, die von Online-Kommunikation mit unbekannten Chatpartnern ausgehen können, bekannt. CyberGrooming und damit einhergehende Manipulationsversuche sowie sexuelle Absichten werden von den Kindern jedoch nicht erwähnt. Weder der Fachbegriff des Cyber-Groomings noch mögliche Strategien zum Vertrauensaufbau werden thematisiert.

\section{Anonymität und Identität im Internet}

Anonymität und Identität spielt bei der Online-Kommunikation eine bedeutende Rolle. Nicht nur die Verschleierung der eigenen Persönlichkeit, auch bewusste Manipulation von Internetprofilen ist online möglich (vgl. Wachs 2017, S. 156). Um zu erkennen, wie Grundschüler*innen bei der Online-Kommunikation auf Profilangaben reagieren, trifft die Handpuppe auf ein informationsgespicktes, ihr allerdings unbekanntes User-Profil. Die Angaben des Accounts setzen sich aus dem Usernamen Lena2010 sowie einem Profilbild eines Mädchens mit braunen Haaren und rotem Kleid zusammen.

Bei der Konfrontation der Handpuppe mit einem unbekannten User-Account steht die Hälfte der befragten Grundschüler*innen diesem von Beginn an skeptisch gegenüber. Die 
wahre Identität, die hinter dem User-Profil vermutet wird, ist bei den kritischen Grundschüler*innen eindeutig negativ geprägt. Die Angaben des Profils werden komplett als Täuschung aufgefasst und ihnen wird kein Vertrauen entgegengebracht. Generell scheint Bewusstsein dafür vorhanden zu sein, dass sämtliche Angaben wie Namen, Geburtsdaten oder Profilbilder keine verlässliche Quelle darstellen und durchaus manipulierbar sind, beispielsweise mithilfe von Bildern aus dem Internet.

Das Pendant dazu schenkt dem User-Account Vertrauen und orientiert sich stark an den Profilangaben. Zwar erkennen auch diese Schüler*innen, dass Rückschlüsse auf wahre Identitäten bei Internetprofilen nie eindeutig möglich sind, dessen ungeachtet vertrauen sie den Profilangaben jedoch zunächst und nutzen diese, um die Identität des Kommunikationspartners näher zu bestimmen. Geschlecht, Alter und Aussehen werden anhand der gegebenen Profilinformationen abgeleitet. Aufgrund des Frauennamens Lena vermuten die Grundschüler*innen hinter der Profilidentität eine weibliche Person. Das Profilbild eines Mädchens mit rotem Kleid und langen Haaren trägt ebenfalls zu dieser Einschätzung bei. Der User-Account wird zudem als gleichaltrig eingestuft. Hinter der Jahresangabe 2010 vermuten diese Schüler*innen das Geburtsjahr des Users, anhand dessen das Alter auf neun Jahre bestimmt wird.

Eine Schülerin konkretisiert ihre Vorstellung der äußeren Erscheinung und beschreibt den Kommunikationspartner, indem Rückschlüsse von Profilbild auf die tatsächliche Identität gezogen werden. Vermutet wird daher eine brünette Haarfarbe und ein rotes Kleid.

Zusammenfassend kann festgehalten werden, dass sich die Hälfte der befragten Schüler*innen von den Profil- und Bildangaben der Chatidentität täuschen ließ. Dennoch standen Schüler*innen diesen Angaben auch kritisch gegenüber und zeigten eine skeptische Grundeinstellung im Umgang mit Anonymität und Identität im Internet.

\section{Umgang mit sensiblen Daten}

Im nächsten Schritt des Interviews galt herauszufinden, auf welche Art und Weise die Schüler*innen mit persönlichen und sensiblen Daten umgehen. Der Handpuppe wird dazu zunächst die Aufforderung gesendet, ihren privaten Wohnort anzugeben. Darauf folgend wird die Übersendung eines Bilds in Unterwäsche gefordert.

Der Weitergabe von persönlichen Daten stehen die befragten Grundschüler*innen kritisch gegenüber. Die Hälfte der Schüler*innen schätzt sensible Kontaktdaten wie Adressen oder Telefonnummern laut eigener Aussage als bedenklich ein und würde diese in Online-Kommunikationssettings nicht preisgeben. Auch die Offenlegung des Nachnamens und die Weitergabe von sensiblem Bildmaterial wird von den Grundschüler*innen kritisch gesehen. Ein Schüler präzisiert diese Aussage und betont, auch eigene Bilder, die sich nicht eindeutig auf die Identität des Schülers rückführen lassen (beispielsweise Bilder ohne Gesicht), nicht zu versenden. Zusätzlich wird erwähnt, dass nicht ausschließlich die Weitergabe von persönlichen Daten in Online-Kommunikationssettings als bedenklich anzusehen ist, sondern auch die generelle Veröffentlichung von Profilinformationen innerhalb Sozialer Netzwerke risikoreich zu bewerten ist und daher mit Vorsicht behandelt werden muss.

Als unbedenklich schätzen die Grundschüler*innen die Angabe des eigenen Namens und des Alters ein. $\mathrm{Ob}$ in diesem Fall der Vor- oder Nachname gemeint ist, wird nicht von allen Teilnehmer*innen präzisiert. Auch der Austausch über alltägliche Themen des Schulalltags oder Treffpunktvereinbarungen mit Freunden werden nicht als Risiko angesehen. Ein Schüler schätzt die Weitergabe von sensiblem Bildmaterial als unbedenklich ein, wenn diese innerhalb des Freundeskreises geschieht. 
Bei Betrachtung der Ergebnisse muss beachtet werden, dass die von den Schüler*innen genannten Verhaltensweisen im Interview-Setting möglicherweise nicht mit Verhaltensweisen im real eintretenden Falle einer Konfrontation mit Cyber-Grooming korrelieren. Diese Annahme kann insbesondere damit begründet werden, dass einzelne Schüler*innen der Weitergabe eigener persönlicher Daten kritisch gegenüberstehen, der Puppe im Setting der Interview-Situation allerdings gegensätzliche Ratschläge geben.

\section{Handlungsstrategien bei risikoreicher Online-Kommunikation}

Sowohl bei der Nachfrage nach Angabe des Wohnortes als auch bei der Forderung eines sensiblen Bildes sollten Verhaltensweisen der Grundschüler*innen in risikoreichen OnlineKommunikationssettings erfasst werden. In folgendem Abschnitt werden die geschilderten Handlungsstrategien der Schüler*innen dargestellt.

\section{Blockieren des Chatpartners}

Um die Online-Kommunikation zu beenden und den Kontakt mit dem Chatpartner abzubrechen, erwähnt ein Schüler die Verwendung des Blockieren-Buttons. Diese Möglichkeit des Kontaktabbruches thematisiert der Grundschüler bereits bei Nachfrage nach dem eigenen Wohnort. Aus den Aussagen des Schülers geht hervor, dass dieser sowohl Kenntnis über die Funktion des Blockierens besitzt als auch (vermutlich) Courage hat, diese zu nutzen. Das Blockieren wird lediglich von einem Grundschüler explizit angesprochen

\section{Löschen des Chats}

Neben dem Blockieren des Kommunikationspartners wird auch das Löschen des Chats von einem Drittel der Grundschüler*innen als mögliche Handlungsstrategie in Betracht gezogen. Die Löschfunktion hat im Unterschied zur Blockier-Funktion auf den meisten Plattformen allerdings keinen dauerhaften Charakter, sondern entfernt den Nachrichtenverlauf lediglich zeitweise von der Bildschirmfläche des Betroffenen. Oft besitzt der Kommunikationspartner trotzdem weiterhin die Möglichkeit, den Betroffenen zu kontaktieren.

Die Strategie des Löschens wird in der Befragung früh angesprochen und identisch zur Blockier-Funktion bereits bei Aufforderung der Wohnortangabe genannt. Ob den Schüler*innen der Unterschied zwischen Blockier- und Löschfunktion bewusst ist, kann nicht näher bestimmt werden.

\section{Aufrechterbaltung des Kontaktes}

Auffällig ist, dass die Mehrheit der befragten Schüler*innen Verhaltensweisen nennt, die die Online-Kommunikation durch Nachfragen oder Antworten, die ein Nachhaken des Kommunikationspartners provozieren, aufrechterhalten. Die Handlungsstrategien führen somit nicht zum Beenden der Kommunikation. Erwähnt werden muss, dass die Aufrechterhaltung des Kontaktes von den Kindern nicht gewollt sein muss. Diese Verhaltensweisen können aus dem Grund resultieren, dass die Grundschüler*innen scheinbar keine alternativen Handlungsstrategien besitzen oder den Kontakt nicht als risikoreich einschätzen.

Eine alternative Verhaltensweise der Aufrechterhaltung des Kontaktes stellt auch das Nachfragen dar. Durch Fragen versuchen die Grundschüler*innen, die Identität oder die Absicht des Chatpartners näher zu bestimmen. Die Nachfragen richten sich dabei gezielt an den Kommunikationspartner. Ob die Schüler*innen Nachfragen stellen, da sie die wahre Intention des Kommunikationspartners nicht selbst beurteilen können und eine konkrete Antwort erwarten 
oder ob diese gestellt werden, um die eigene Einschätzung der Risikosituation zu untermauern, wurde während der Interview-Situation nicht detaillierter in den Blick genommen.

Auch werden verschleiernde Verhaltensweisen dargelegt, die zwar keine direkten Nachfragen darstellen, den Kommunikationspartner jedoch zu weiteren Antworten animieren. Identisch zur Strategie des Nachfragens wird auch an dieser Stelle kein Kommunikationsabbruch erzielt. Bei Nachfrage nach dem eigenen Wohnort antworten die Schüler*innen mit der Anpreisung eines Geheimnisses. Auch die Nutzung von Aussagen, die Unschärfe ausdrücken, beispielsweise die Antwort „Vielleicht“, regen den Kommunikationspartner zu weiteren Nachfragen an.

Ein Schüler äußerte die Nennung einer falschen Adresse. Er versucht auf diese Weise, konkrete Angaben zu vermeiden, die Online-Kommunikation wird durch diese Strategie allerdings nicht explizit abgebrochen, sondern weiter aufrechterhalten.

Eine weiterführende Form der Aufrechterhaltung des Kontaktes stellt das Initiieren eines persönlichen Treffens dar. Auf die Aufforderung, den eigenen Wohnort anzugeben, schlägt ein Schüler eine Verabredung im Park vor. Der Kontakt wird durch den Vorschlag eines Treffens in der Offline-Welt nicht nur aufrechterhalten, sondern bei tatsächlicher Umsetzung des Vorschlages sogar intensiviert. Grundsätzlich muss überlegt werden, ob der Schüler durch das Vorschlagen der Verabredung im Park evtl. eine zuvor erlernte Handlungsstrategie anwendet, die das Treffen mit Internetbekanntschaften an öffentlichen Plätzen empfiehlt.

\section{Bestimmt abwehren}

Zwei der befragten Grundschüler*innen reagieren auf die Aufforderung, ein sensibles Bild zu versenden, mit der Ablehnung dieser Bitte. Die Viertklässler*innen formulieren deutlich, dass der Forderung des Chatpartners nicht nachgegangen wird. Auch an anderen Stellen der Online-Kommunikation drücken die Grundschüler*innen deutliche Ablehnung gegenüber der Kommunikation aus. Eine eigene anschließende Handlungsstrategie, die zum Abbruch des Kontaktes führt (beispielsweise das Blockieren des Kommunikationspartners), erwähnt jedoch lediglich ein Schüler im Anschluss.

\section{Ignorieren}

Das Ignorieren der Nachrichten des Kommunikationspartners wird von einem Schüler erwähnt. Durch das Nicht-Antworten auf die Kommunikationsversuche des Chatpartners wird diesem signalisiert, dass weitere Kommunikation nicht gewünscht ist. Auffallend ist, dass der Schüler bereits bei Kontaktaufnahme vonseiten des Kommunikationspartners daran appellierte, diesem nicht zu antworten.

\section{Hilfe durch Außenstehende}

Die Unterstützung von außenstehenden Bezugspersonen kann Grundschüler*innen helfen, in unangenehmen Online-Situationen angemessen zu agieren und Kommunikationssettings somit zielführend zu beenden. Welche Ansprechpersonen die Grundschüler*innen während des Leitfadeninterviews in Erwägung ziehen, wird in folgendem Abschnitt dargestellt.

\section{Nabe Verwandte und Freunde}

Während der Interview-Simulation nennen zwei Drittel der Schüler*innen als erste Ansprechperson in unangenehmen Online-Situationen die Eltern. Ein Schüler konkretisiert diese Angabe und nennt explizit seine Mutter, die ihm in der risikoreichen Situation weiterhelfen kann. 
Alle Grundschüler*innen, die einen älteren Bruder besitzen, würden auch diesen um Hilfe bitten. Weitere Geschwisterkinder werden nicht erwähnt. Auch geben die Grundschüler*innen an, sich an Freund*innen zu wenden, denen sie vertrauen.

Prinzipiell ziehen die Schüler*innen während der Interview-Situation nicht von selbst in Erwägung, eine dritte Person um Hilfe zu beten. Erst auf Nachfrage und dem damit gegebenen Gedankenimpuls vonseiten der Interview-Leiterin nennen die Viertklässler*innen oben angeführte Ansprechpersonen. Lediglich ein Schüler rät ohne vorherigen Impuls der Puppe Clara, die unangenehme Situation mit Hilfestellung eines Erwachsenen zu lösen.

Polizei

Die Polizei wird ebenfalls als mögliche Anlaufstelle in unangenehmen Online-Situationen genannt.

Auffällig ist, dass eine Schülerin beschreibt, die Polizei erst in Kenntnis zu setzen, wenn sich die Kommunikation verschlimmert. Eine detaillierte Vorstellung dieser Verschlimmerung wird nicht formuliert.

\subsection{Typologisierung}

Anhand der dargestellten Ergebnisse war es möglich, die Grundschüler*innen auf Grundlage ihrer Handlungsstrategien in drei Handlungstypen zu kategorisieren. Diese können für weiterführende Forschung interessant sein.

Die Handlungstypen beschreiben Gemeinsamkeiten, die die befragten Grundschüler*innen beim Umgang mit risikoreicher Online-Kommunikation auszeichnete. Die erforschten Ansätze von Wachs, Wolf und Pan (2012) sind auch in vorliegender Studie erkennbar. Daher sind Übereinstimmungen möglich. Grundsätzlich handelt es sich um Pauschalisierungen, die aufgrund der vorliegenden Forschung gezogen werden konnten. Die Handlungstypen werden im folgenden Abschnitt erläutert.

\section{Der kritisch-abwehrende Typ}

Kritisch-abwehrende Grundschüler*innen stehen der Online-Kommunikation mit unbekannten Chatpartnern skeptisch gegenüber. Aus diesem Grund lassen sie sich von Profilbildern und -angaben nicht überzeugen, sondern stellen diese infrage. Über die Möglichkeit der Manipulation von Profilinformationen wissen die Grundschüler*innen Bescheid und kennen explizite Strategien, mit denen Informationen verfälscht werden können.

Bei Kontaktaufnahme in Online-Kommunikationssettings reflektieren die Grundschüler*innen mögliche Intentionen des Chatpartners und beziehen auch solche mit ein, die für sie selbst gefährdend sein könnten. Generell kennen die Grundschüler*innen mögliche Gefahren und Risiken des Internets, auch wenn die Gefahr des Cyber-Groomings nicht explizit genannt wird.

Aufgrund der kritischen Einstellung gegenüber anonymen Kommunikationspartnern und des Bewusstseins für mögliche Gefahren der Online-Kommunikation versuchen die Grundschüler*innen, den Nachrichtenverkehr bereits nach Kontaktaufnahme zu beenden. Hierbei wird zuerst auf das Ignorieren zurückgegriffen, bei anhaltendem Kontakt allerdings keine Scheu vor dem Einsatz des Blockieren-Buttons gezeigt. Die Schüler*innen kennen Anlaufstellen, an die sie sich bei Ungewissheit wenden können.

Mit eigenen persönlichen Daten gehen Grundschüler*innen des kritisch-abwehrenden Typs vorsichtig um. Nicht nur die Weitergabe dieser Informationen, auch die grundsätzliche Angabe von Profilinformationen auf Plattformen wird abgelehnt. 
Innerhalb der Stichprobe konnte lediglich ein Schüler diesem Typ zugeordnet werden. Die erläuterten Merkmale beruhen daher ausschließlich auf den Aussagen dieses Schülers. Da sich seine Handlungs- und Verhaltensweisen jedoch grundsätzlich von den anderen Typen unterscheiden, konnte ein eigener Typus generiert werden.

\section{Der zögerlich-binterfragende Typ}

Identisch zum kritisch-abwehrenden Typ stehen auch Grundschüler*innen des zögerlichhinter-fragenden Typs Internetkontakten und Profilangaben kritisch gegenüber. Sie ziehen in Betracht, dass sich hinter Internet-Profilen eine andere Person als angegeben verbergen könnte.

Gefahren und Risiken der Online-Kommunikation sind den Schüler*innen vereinzelt bewusst, sodass diese grundsätzlich mit Vorsicht in Kommunikationssettings agieren. Die Intention der Kontaktaufnahme wird als bösartig angesehen. Dabei wird in den Mittelpunkt gestellt, ein persönliches Treffen zu vereinbaren oder Einbruch zu begehen.

Auch mit eigenen sensiblen Daten gehen Grundschüler*innen des zögerlich-hinterfragenden Typs vorsichtig um und geben an, keine persönlichen Informationen an unbekannte Kommunikationspartner weiterzugeben. Bei Handlungsabfrage wird jedoch deutlich, dass sie die Forderung nach persönlichen Angaben nicht unmittelbar abwehren, sondern die Kommunikation ungewollt durch ablenkende und unpräzise Antworten aufrechterhalten.

Generell hinterfragen die Grundschüler*innen die Absichten des Kommunikationspartners, indem sie konkrete Fragen stellen, um Intentionen offenzulegen oder nähere Informationen zur Identität erhalten. Das explizite Beenden der Kommunikationssituation wird nicht in Erwägung gezogen. Auf Nachfrage des Interviewers können die Grundschüler*innen Ansprechpartner wie die Eltern oder die Polizei nennen.

\section{Der naiv-bilflose Typ}

Die Grundschüler*innen des naiv-hilflosen Typs stehen Internetkontakten in geringem Maße kritisch gegenüber. Anhand von Profilangaben und Profilbildern wird versucht, Schlussfolgerungen zu ziehen, die auf die Identität des Kommunikationspartners schließen lassen. Die Grundschüler*innen verlassen sich stark auf die im Internet angegebenen Informationen.

Vereinzelte Gefahren und Risiken der Online-Kommunikation können die Grundschüler*innen des naiv-hilflosen Typs nennen. Ähnlich wie bei den bereits erläuterten Typen versuchen die Grundschüler*innen mit persönlichen Daten schützend umzugehen und können bedenkliche Angaben nennen, die nicht weitergegeben werden sollten. Der Ratschlag an die Handpuppe fällt - auch wenn dieser im Nachhinein überdacht und widerrufen wird - allerdings anders aus. In dieser Situation handeln die Schüler*innen fahrlässig und stimmen der Datenweitergabe ohne nähere Überlegungen zu.

Auch Handlungsstrategien, die den Kontakt zum Kommunikationspartner endgültig beenden, werden nicht genannt. Ferner wird auf den Chatpartner eingegangen und die Kommunikation aufrechterhalten, indem konkretes Interesse gezeigt wird. Eine genannte Handlungsstrategie, die den Kontakt zumindest zeitweise einschränkt, stellt das Löschen dar. Diese wird allerdings nicht von allen Grundschüler*innen des Typus angesprochen. Auf Nachfrage können die Schüler*innen entsprechende Ansprechpartner nennen, die bei unangenehmer Online-Kommunikation kontaktiert werden können. 
Deutlich wird, dass die Grundschüler*innen des naiv-hilflosen Typs kommunikativen Online-Settings offen gegenüberstehen und unerfahren handeln. Mögliche Folgen und entsprechend zielverfehlende Handlungsweisen werden erst spät erkannt.

\section{Präventionsmaßnahmen zum Schutz vor Cyber-Grooming}

Ziel der Forschungsarbeit war es, Präventionsmaßnahmen zum Schutz vor Cyber-Grooming für Schüler*innen der Grundschule auf der Grundlage deren Handlungsstrategien herauszuarbeiten. Im Folgenden werden daher entsprechende Maßnahmen von Präventionskonzepten fokussiert, die durch Erkenntnisse der dargestellten Handlungsstrategien herausgearbeitet werden konnten.

\section{Aufklärungs- und Sensibilisierungsarbeit}

Im Sinne einer umfassenden Medienkompetenzförderung ist es unentbehrlich, Grundschüler*innen mit Gefahren und Risiken der digitalen Medien vertraut zu machen (vgl. Rauh 2016, S. 71).

Die Schüler*innen erwähnen Cyber-Grooming im Verlauf des Interviews nicht. Dies lässt darauf schließen, dass sie mit dieser Gefahr nicht vertraut sind oder diese im Theaterspielsetting nicht in Erwägung ziehen. Da selbst bei der Aufforderung, ein intimes Bild zu versenden, sowohl die Gefahr des Cyber-Groomings als auch mögliche sexuelle Absichten des Chatpartners nicht erwähnt werden, ist es notwendig, Aufklärungs- und Sensibilisierungsarbeit zu leisten. Auf diese Weise können Kinder den Tatvorhergang erkennen und je nach Ausgangslage entsprechende Handlungsstrategien anwenden.

Insbesondere müssen bei der Aufklärungs- und Sensibilisierungsarbeit manipulative Vorgehensweisen der Täter*innen in den Blick genommen werden, um Kindern das Erkennen der Strategien zu ermöglichen. Dass die Täter*innen zu Beginn der Kommunikation stets freundlich und zuvorkommend wirken, prinzipiell jedoch andere Ziele verfolgen, muss den Grundschüler*innen bewusst sein.

Beachtet werden muss, dass bei der Bereitstellung von Informationen zwar Gefahren verdeutlicht werden sollen, allerdings zu keinem Zeitpunkt Angst oder Panik generiert werden darf. Entsprechende Inhalte wie grenzüberschreitende Fragen der Täter*innen müssen den Schüler*innen daher mit Vorsicht präsentiert werden.

Ziel einer umfassenden Medienkompetenzförderung ist es, Schüler*innen zur selbstsicheren und furchtfreien Nutzung digitaler Medien zu verhelfen. Daher muss den Grundschüler*innen ihr durch die Aufklärung erlangter Expertenstatus verdeutlicht werden (vgl. Enders 2004, S. 17). Auf diese Weise können Schüler*innen beim selbstbewussten Umgang mit digitalen Medien und Online-Kommunikation unterstützt werden.

\section{Anonymität im Internet}

Um Prävention in Bezug auf Cyber-Grooming zu leisten, ist es bedeutsam, Anonymität und Identität im Internet sowie damit einhergehende Risiken anzusprechen. Die Schüler*innen müssen ein Bewusstsein dafür entwickeln, dass niemals eindeutig sichergestellt werden kann, wer sich hinter Internet-Profilen verbirgt (vgl. Weißer Ring e. V. 2019, o. S.).

Die vorliegenden Ergebnisse haben gezeigt, dass die Mehrheit der Schüler*innen anonymen Profilen grundsätzlich kritisch gegenübersteht. Trotzdem verlassen sich Grundschüler*innen auf Profilangaben und schenken diesen Vertrauen. Die mögliche Manipulation von Profilbildern und weiteren Informationen muss daher bei entsprechenden Präventionseinheiten 
thematisiert werden. Bei Kommunikation mit unbekannten Chatpartnern sollte aus diesem Grund stets mit Vorsicht und Reflexion gehandelt werden.

\section{Umgang mit sensiblen Daten}

Anonymität im Internet kann auch vorteilhaft zum eigenen Schutz eingesetzt werden. Da Cyber-Grooming-Täter*innen persönliche Informationen der Kinder für Auswahl- oder Manipulationsstrategien nutzen, müssen sensible Daten sowohl bei der Veröffentlichung in Sozialen Netzwerken als auch bei der Weitergabe in Online-Kommunikationssettings stets mit Vorsicht behandelt werden. Die Schüler*innen müssen die Bedeutsamkeit des achtsamen Umgangs mit privaten Angaben verinnerlichen (vgl. Weißer Ring e. V. 2019, o. S.).

Die Gestaltung des eigenen Online-Profils in Sozialen Netzwerke darf nicht darauf schließen lassen, wer sich dahinter verbirgt (vgl. Weißer Ring e. V. 2019, o. S.). Thematisiert werden sollte zudem, dass ein mit Informationen gespicktes Profil die Gefahr erhöht, von CyberGrooming betroffen zu werden (vgl. Wachs 2017, S. 321). Da es angesichts der starken Nutzung von Social Media-Anwendungen nur bedingt realitätsnah erscheint, den heranwachsenden Schüler*innen den Bild-Upload gänzlich zu verbieten, muss zumindest auf entsprechende Privatsphäre- und Profileinstellungen verwiesen werden. Mithilfe dieser kann die Öffentlichkeit der Informationen eingeschränkt und eine erste Hürde für Cyber-GroomingTäter*innen geschaffen werden.

Bei Betrachtung der vorliegenden Ergebnisse muss insbesondere der Umgang mit persönlichen Daten in Chatsituationen geschult werden. Lehrpersonen müssen an dieser Stelle verdeutlichen, dass sensible Informationen wie Telefonnummern oder Adressen niemals weitergegeben werden dürfen. Auch wenn der Chatpartner Geheimhaltung der Daten versichert, muss den Schüler*innen bewusst sein, dass die Weitergabe von Bild- und Videomaterial Risiken birgt und davon abgesehen werden sollte.

\section{Beenden der Kommunikation}

Neben der Aufklärungs- und Sensibilisierungsarbeit ist es essenziell, den Schüler*innen passende Handlungsstrategien nahezulegen, mit denen Anbahnungsversuche von Täter*innen abgewehrt werden können (vgl. Katzer 2014, S. 182). Durch die vorliegenden Untersuchungsergebnisse wurde deutlich, dass Handlungsstrategien, die zum Abbruch der Kommunikation führen, nicht bei allen Schüler*innen vorhanden sind. Um den Kontakt zielführend zu beenden, ist es sinnvoll, den Chatpartner zu blockieren (vgl. Weißer Ring e. V. 2019, o. S.). Den Schüler*innen muss dazu einerseits die technische Funktion vorgestellt und erläutert werden, andererseits muss verdeutlicht werden, dass das Blockieren eines Kommunikationspartners jederzeit erlaubt ist. Bei Betrachtung der dargestellten Ergebnisse ist zudem die Thematisierung bedeutsam, dass das von den Grundschüler*innen genannte Löschen des Chatpartners möglicherweise nicht dieselbe Effektivität wie eine Blockierung erzielt.

Generell ist es im Sinne von zielführenden Handlungsstrategien relevant, Schüler*innen zum Abbruch des Kontaktes anzuregen. Cyber-Grooming-Täter*innen sollte keine Möglichkeit gegeben werden, die Schüler*innen durch Manipulation zu beeinflussen und weiterhin in die Unterhaltung einzubinden.

\section{Stärkung des eigenen Selbstbewusstseins}

Eine bedeutende Rolle bei Präventionsmaßnahmen spielt die Stärkung des eigenen Selbstwertgefühls. Insbesondere jüngeren Kindern muss verdeutlicht werden, dass sie ihren Gefühlen trauen können, wenn ihnen eine Situation seltsam erscheint (vgl. Freelance 2018, S. 7). 
Prävention sollte daher auch am Selbstvertrauen der Grundschüler*innen ansetzen und dazu ermutigen, eigene Grenzen klar zu machen (vgl. Wachs 2017, S. 322). Eine Verknüpfung mit der im Idealfall in Schulen bestehenden Sexualpädagogik ist sinnvoll, um bei auch bei grenzüberschreitenden Inhalten im Internet selbstbestimmt agieren zu können (vgl. Wanzek-Sielert 2002, S. 538).

Generell muss sowohl bei der Aufklärungs- und Sensibilisierungsarbeit als auch bei der Thematisierung von Handlungsstrategien in Online-Kommunikationssettings zu jeder Zeit verdeutlicht werden, dass die Grundschüler*innen keine Schuld tragen, wenn der Kontakt trotz entsprechender Abwehrversuche weiterhin existiert oder Täter*innen sie erneut kontaktieren (vgl. Buskotte 2001, S. 62). Auch muss betont werden, dass die Schuld selbst bei Missachtung der Präventionsmaßnahmen immer bei den Täter*innen und niemals bei den Betroffenen liegt (vgl. UBSKM 2019).

\section{Anschlusskommunikation}

Neben eigenen Handlungsstrategien ist die Vermittlung von Anlaufstellen relevant, an die sich die Schüler*innen und Schüler bei Unsicherheiten wenden können. Auch im Falle einer erneuten Kontaktaufnahme der Täter*innen können Ansprechpersonen Hilfe leisten. Die Vertrauensperson muss den Grundschüler*innen in allen Phasen des Cyber-Groomings entsprechende Hilfe anbieten. Schüler*innen sollten nicht das Gefühl vermittelt bekommen, sich erst bei tatsächlicher Viktimisierung an Außenstehende wenden zu dürfen (vgl. Vogelsang 2017, S. 162f.).

Es wird deutlich, dass sich die Grundschüler*innen bevorzugt an Ansprechpartner innerhalb der Familie wenden. Prinzipiell ist dieses Verhalten zielführend, wenn die Familienmitglieder über ausreichend Medienkompetenz verfügen, um den Schüler*innen effektiv weiterzuhelfen, und sollte daher gefördert werden. Gleichzeitig ist es bedeutsam, den Grundschüler*innen auch außerfamiliäre Anlaufstellen wie Hilfsorganisationen oder Lehrpersonen nahe zu legen, die kontaktiert werden können, wenn zu den eigenen Eltern und/oder Geschwistern kein gutes Verhältnis besteht oder diese keine geeignete weiterführende Hilfe anbieten können.

Auch können die Schüler*innen mit rechtlichen Vorgehensweisen vertraut gemacht werden (vgl. Mathiesen 2014, S. 31). Die Kontaktaufnahme zur Polizei und das Stellen einer Strafanzeige ist bei sexueller Online-Belästigung sinnvoll. Daher sollte den Grundschüler*innen möglicher Respekt und unnötige Scheu vor diesem Schritt genommen werden (vgl. Mathiesen 2014, S. 31), indem die Vorgehensweise erläutert und auf diese Weise Transparenz geschaffen wird.

\section{Peer-to-Peer-Education}

Bei Einsatz von entsprechenden Präventionsmaßnahmen kann die zurzeit noch selten umgesetzte, aber erfolgversprechende Peer-to-Peer-Methode in Betracht gezogen werden (vgl. Katzer 2014, S. 184). Peer-to-Peer-Education bezeichnet die Aufklärungs- und Präventionsarbeit von Kindern durch Gleichaltrige (vgl. Katzer 2014, S. 184). Vorteile bietet nicht nur das Vertrauens- und Wertschätzungsverhältnis, das Schüler*innen den sogenannten Scouts entgegenbringen, sondern auch die Entlastung von Lehrpersonen, wenn medienpädagogische Förderung zumindest in Teilbereichen von Scouts übernommen wird (vgl. Katzer 2014, S. 184).

Da der „generationslogische Prozess“ (Friedrichs/Sander 2010, S. 284) der Weitergabe von Wissen von älteren an jüngere Generationen bei Medienkompetenz nicht mehr konsequent umgesetzt wird, sondern Kompetenzen und Nutzungshinweise auch innerhalb der Peers ausgetauscht werden (vgl. Friedrichs/Sander 2010, S. 284), wäre es sinnvoll, diesen Prozess gezielt 
zu unterstützen. Inwiefern das Konzept bereits in die Grundschule eingebunden werden kann, bedarf weiterer Forschung. Denkbar ist insbesondere der Einsatz von älteren Jugendlichen als Scouts.

\section{Resümee}

\section{Zusammenfassung der Ergebnisse}

Die Ergebnisse der Untersuchung haben gezeigt, dass digitale Medien und damit einhergehende Möglichkeiten der Online-Kommunikation von Schüler*innen der Grundschule in erheblichem Maße genutzt werden. Zum größten Teil ist diese Mediennutzung autonom geprägt. Deutlich wurde, dass Präventionsmaßnahmen, die Grundschüler*innen mit Gefahren und Risiken des Internets vertraut machen, unentbehrlich sind.

Mit dieser Arbeit sollten Einblicke in Handlungsstrategien von Grundschüler*innen bei risikoreicher Online-Kommunikation ermöglicht werden. Auf der Grundlage dieser Erkenntnisse sollten Präventionsmaßnahmen zum Schutz vor Cyber-Grooming konzipiert werden. Prinzipiell ist zu sagen, dass alle befragten Schüler*innen zumindest über vereinzelte Gefahren und Risiken der Online-Kommunikation informiert sind. Das explizit in vorliegender Arbeit zu untersuchende Cyber-Grooming wurde allerdings von keinem Grundschüler*in thematisiert. Die Handlungsstrategien der Grundschüler*innen in den angesprochenen Risikosituationen sind unterschiedlich gestaltet. Auffällig ist, dass die Mehrheit der befragten Schüler*innen in Kommunikationssettings, die eine Gefahr darstellen können, nicht zielführend reagiert und zum Teil leichtsinnig handelt.

Die gestaltungsorientierte Forschungsfrage ermöglichte es, Maßnahmen zum Schutz vor Cyber-Grooming zu formulieren. Zusammenfassend werden Präventionskonzepte benötigt, die Cyber-Grooming als Gefahr anerkennen und dementsprechende Aufklärungs- und Sensibilisierungsarbeit leisten. Den Schüler*innen muss die Relevanz eines reflektierten Umgangs im Internet nahegelegt werden. Ferner müssen Handlungsstrategien vermittelt werden, die Cyber-Grooming zielführend abwehren können.

\section{Diskussion der Ergebnisse}

Die Forschungsfrage der vorliegenden Arbeit konzentrierte sich auf die Gestaltung von Präventionsmaßnahmen zum Schutz vor Cyber-Grooming, die in der Grundschule eingesetzt werden können. Cyber-Grooming stellt ein weitgehend unerforschtes Phänomen dar. Es ist zu erwarten gewesen, dass Grundschüler*innen mit dieser Thematik noch nicht vertraut sind. Die hier zusammengefasste Forschungsarbeit konnte bestätigen, dass Schüler*innen CyberGrooming nicht kennen oder diese zumindest im gewählten Forschungssetting nicht als relevant angesehen haben.

Die Relevanz von Medienkompetenzförderung bestätigte sich zudem in Hinblick auf die umfassende Mediennutzung der befragten Grundschüler*innen. Nach Betrachtung der Ergebnisse der KIM-Studie (MPFS 2018) ist zu erwarten gewesen, dass auch die Grundschüler*innen der vorliegenden Studie bereits mit digitalen Medien in Berührung gekommen sind. Bei Auswertung des Kurzfragebogens wird deutlich, dass die befragten Grundschüler*innen Medien und Internet intensiv nutzen und auch Plattformen, die für ihre Altersklasse noch nicht vorgesehen sind, bereits verwenden.

An dieser Stelle muss beachtet werden, dass die Mediennutzung der befragten Grundschüler*innen möglicherweise übereinstimmend intensiv ausfällt, da diese dieselbe Schulklasse 
besuchen und somit evtl. in derselben Peer-Group aufwachsen. Eine gegenseitige Beeinflussung bezüglich Nutzung verschiedener Medien und Plattformen ist denkbar. Trotzdem wäre in Hinblick auf diese umfassende Nutzung zu erwarten gewesen, dass die Schüler*innen bereits vielfältige Handlungsstrategien bei Konfrontation mit Gefahren im Internet besitzen. Die hier vorgestellte Studie konnte dies nicht bestätigen.

Insgesamt kann festgestellt werden, dass die Mehrheit der Grundschüler*innen Typen zugeordnet werden konnte, die Cyber-Grooming nicht zielführend abwehren können. Neben dem naiv-hilflosen Typ, der aufgrund seiner fehlenden Handlungsstrategien einem erhöhten Risiko der Viktimisierung ausgesetzt ist, ist auch der zögerlich-hinterfragende Typ durch die Aufrechterhaltung der Kommunikation gefährdet, von Cyber-Grooming-Täter*innen in weitere Kommunikation verwickelt zu werden.

Warum der Handpuppe in manchen Aspekten - im Vergleich zu den von Grundschüler*innen angesprochenen, zum eigenen Schutz zielführenderen Handlungsweisen - abweichende Anweisungen erteilt werden, ist unklar. Denkbar wäre, dass die Ratschläge an die Handpuppe eine erste spontane Reaktion darstellen und erst im Verlauf des Interviews bei gemeinsamem Austausch näher über Gefahren und dementsprechende Verhaltensweisen nachgedacht wird. Eine andere Möglichkeit stellt die Betrachtung der Theaterspiel-Situation zu Unterhaltungszwecken der Grundschüler*innen dar. Da die Viertklässler*innen sich bewusst sind, dass der Kommunikationsverlauf simuliert ist und die Handpuppe keinen Schaden davon trägt, ist denkbar, dass die Schüler*innen kontroverse Antworten geben, um spannendere Unterhaltung zu erfahren.

Grundsätzlich muss bei Betrachtung der Ergebnisse beachtet werden, dass die hier beschriebene Forschungsarbeit keine Repräsentativität oder Vollständigkeit gewährleisten kann. Dennoch war es möglich, anhand der Einblicke medienpädagogische Konsequenzen zu formulieren.

\section{Medienpädagogische Konsequenæen und Ausblick}

Die Typologisierung hat gezeigt, dass in Grundschulklassen bei der Thematisierung von Gefahren und Risiken im Internet kein homogener Wissens- und Handlungsstand erwartet werden darf. Präventionsmaßnahmen zum Schutz vor Cyber-Grooming müssen daher die gewonnenen Erkenntnisse der Typenbildung mit einbeziehen und sowohl Grundschüler*innen fördern, die über zielführende Handlungsstrategien verfügen, als auch medienunerfahrenen Schüler*innen Hilfeleistung und Unterstützung bieten. Inwiefern in diesem Sinne auch die erläuterte Peer-to-Peer-Methode einzusetzen ist, muss je nach Klassenstufe und Umsetzungsmöglichkeit individuell beurteilt werden.

$\mathrm{Da}$ auch in vorliegender Studie bereits ein Grundschüler Kontakt zu einem unbekannten Kommunikationspartner hatte, muss insbesondere bei Präventionsmaßnahmen zu sensiblen Themen wie Cyber-Grooming beachtet werden, dass sich unter den Teilnehmer*innen Betroffene befinden können (vgl. Vogelsang 2017, S. 336). Ein sensibler Umgang sowie Angebote von Hilfestellungen sind daher verpflichtend (vgl. Vogelsang 2017, S. 337).

Trotz Umsetzung der erläuterten Präventionskonzepte darf nicht außer Acht gelassen werden, dass selbst im Umgang mit den Medien versierte und selbstbewusste Kinder den Strategien von erwachsenen Täter*innen unterlegen sind. Zwischen Täter*innen und Betroffenen existiert auch bei wahrgenommenen Präventionsmaßnahmen ein Machtungleichgewicht (vgl. Wachs 2017, S. 50). Identisch zu anderen Präventionskonzepten darf daher auch von schulischer Prävention nicht erwartet werden, dass eine Betroffenheit vollständig verhindert werden kann (vgl. Marquardt-Mau 2002, S. 44). 
Dennoch kann die Grundschule einen erheblichen Teil dazu beitragen, flächendeckende Prävention zu ermöglichen, die sowohl explizite Themenbereiche wie Cyber-Grooming als auch übergreifende Medienbildung in den Blick nimmt. Lehrer*innen können Grundschüler*innen mithilfe von Medienbildung dabei unterstützen, bei eigener Mediennutzung selbstbewusst und kritisch zu agieren. Für die Zukunft wäre daher wünschenswert, dass eine in der Grundschule beginnende, nachhaltige Medienkompetenzförderung umgesetzt wird.

\section{Literatur}

Braun, Gisela (2001): Der Alltag ist sexueller Gewalt zuträglich. Prävention als Antwort auf alltägliche Gefährdungen von Mädchen und Jungen. In: Risau, Petra/Kruck, Marlene/Bender, Kathrin (Hrsg.): Sexualisierte Gewalt in der Alltags- und Medienwelt von Kindern. Wahrnehmen. Benennen. Präventiv handeln. Bad Heilbrunn: Klinkhardt, S. 119-124.

Bullens, Ruud (1995): Der Grooming Prozeß - oder das Planen des Mißbrauchs. In: Marquardt-Mau, Brunhilde (Hrsg.): Schulische Prävention gegen sexuelle Kindesmißhandlung. Grundlagen, Rahmenbedingungen, Bausteine und Modelle. Weinheim und München: Juventa Verlag, S. 55-67.

Buskotte, Andrea (2001): Mama, was ist ein Kinderschänder? Berichte über sexuelle Gewalt in TVNachrichtensendungen - Konsequenzen für die Prävention. In: Risau, Petra/Kruck, Marlene/Bender, Kathrin (Hrsg.): Sexualisierte Gewalt in der Alltags- und Medienwelt von Kindern. Wahrnehmen. Benennen. Präventiv handeln. Bad Heilbrunn: Klinkhardt, S. 57-63.

Dekker, Arne/Koops, Thula/Briken, Peer (2016): Sexualisierte Grenzverletzungen und Gewalt mittels digitaler Medien. Zur Bedeutung digitaler Medien für Phänomene sexualisierter Grenzverletzungen und Gewalt gegen Kinder und Jugendliche. Expertise. Berlin: Arbeitsstab des Unabhängigen Beauftragten für Fragen des sexuellen Kindesmissbrauchs. URL: docs.dpaq.de/117632a_expertise_sexuelle_gewalt_an_kindern_mittels_digitaler_medien.pdf (12.05.2019)

Enders, Ursula (2004): Sexueller Missbrauch in den Chaträumen des Internets. Wie Mädchen und Jungen sexuell ausgebeutet werden und wie Erwachsenen sie davor schützen können. URL: zartbitter.de/0/Eltern_und_Fachleute/5400_ sexueller_Missbrauch_in_den_chatraeumen_des_internets.pdf (23.04.2019)

Freelance (2018): Fachwissen für Lehrpersonen. Neue Medien. Cybergrooming. URL: be-freelance.net/images/freelance/pdf/unterrichtsmodule/digitale_medien/cybergrooming/cybergrooming_fachwissen.pdf (02.06.2019)

Friedrichs, Henrike/Sander, Uwe (2010): Peers und Medien - die Bedeutung von Medien für den Kommunikations- und Sozialisationsprozess im Kontext von Peerbeziehungen. In: Harring, Marius/Böhm-Kasper, Oliver/Rohlfs, Carsten/Palentien, Christian (Hrsg.): Freundschaften, Cliquen und Jugendkulturen. Peers als Bildungs- und Sozialisationsinstanzen. Wiesbaden: VS Verlag für Sozialwissenschaften. S. 283-309.

Gläser, Jochen/Laudel, Grit (2010): Experteninterviews und qualitative Inhaltsanalyse. Wiesbaden: VS. Gottschalk, Petter (2011): A Dark Side of Computing and Information Sciences: Characteristics of Online Groomers. In: Journal of Emerging Trends in Computing and Information Sciences. Vol. 2 Nr. 9, pp. 447-455. URL: cisjournal.org/journalofcomputing/archive/vol2no9/vol2no9_5.pdf (18.05.2019)

Grimm, Petra/Rhein, Stefanie/Clausen-Muradian, Elisabeth (2008): Gewalt im Web 2.0. Der Umgang Jugendlicher mit gewalthaltigen Inhalten und Cyber-Mobbing sowie die rechtliche Einordnung der Problematik. Schriftenreihe der NLM. Berlin: Vistas.

Katzer, Catarina (2007): Gefahr aus dem Netz. Der Internet-Chatroom als neuer Tatort für Bullying und sexuelle Viktimisierung von Kindern und Jugendlichen. URL: kups.ub.unikoeln.de/2152/1/DissertationCatarinaKatzer2007.pdf (18.05.2019)

Katzer, Catarina (2014): Cybermobbing. Wenn das Internet zur W@ffe wird. Berlin: Springer. 
KIM-Studie (2018): KIM-Studie 2018. Kinder, Internet, Medien. Basisuntersuchung zum Medienumgang 6- bis 13-Jähriger. Stuttgart: Medienpädagogischer Forschungsverbund Südwest.

Marquardt-Mau, Brunhilde (2002): Prävention in der Schule. In: Bange, Dirk/Körner, Wilhelm (Hrsg.): Handwörterbuch Sexueller Missbrauch. Göttingen: Hogrefe, S. 438-444.

Mathiesen, Asbjørn (2014): Cybermobbing und Cybergrooming. Neue Kriminalitätsphänomene im Zeitalter moderner Medien. Hannover: Kriminalwissenschaftliches Institut der Leibniz Universität Hannover. URL: repo.uni-hannover.de/bitstream/ handle/123456789/3710/Cybermobbing $\% 20$ und $\% 20$ Cybergrooming.pdf?sequence $=1$ (12.05.2019)

Rauh, Felix (2016): Fit und Fair im Netz. Strategien zur Prävention von Sexting und Cyberbullying. Bern: hep.

Roll, Timo (2017): Cyber-Mobbing als neue Gewalt unter Jugendlichen im digitalen Zeitalter. Medienverhalten, Problemdarstellung und mögliche Handlungsoptionen. Norderstedt: GRIN.

Schreier, Margrit (2004): Qualitative Methoden. In: Mangold, Roland/Vorderer, Peter/Bente, Gary (Hrsg.): Lehrbuch der Medienpsychologie. Göttingen: Hogrefe, S. 377-400.

UBSKM - Unabhängiger Beauftragter für Fragen des sexuellen Kindesmissbrauchs (2019): Cybergrooming. URL: beauftragter-missbrauch.de/ praevention/sexuelle-gewalt-mittels-digitalermedien/cybergrooming (22.04.2019)

Vogelsang, Verena (2017): Sexuelle Viktimisierung, Pornografie und Sexting im Jugendalter. Ausdifferenzierung einer sexualbezogenen Medienkompetenz. Wiesbaden: VS.

Wachs, Sebastian (2017): Gewalt im Netz. Studien über Risikofaktoren von Cyberbullying, Cybergrooming und Poly-Cyberviktimisierung unter Jugendlichen aus vier Ländern. Hamburg: Dr. Kovac.

Wachs, Sebastian/Wolf, Karsten D./Pan, Ching-Ching (2012): Cybergrooming. Risk Factors, Coping Strategies and Associations with Cyberbullying. In: Psicothema, 24(4), pp. 628-633.

Wanzek-Sielert, Christa (2002): Sexualpädagogik. In: Bange, Dirk/Körner, Wilhelm (Hrsg.): Handwörterbuch Sexueller Missbrauch. Göttingen: Hogrefe Verlag, S. 536-542.

Webster, Stephen/Davidson, Julia/Bifulco, Antonia/Gottschalk, Petter/Caretti, Vincenzo/Pham, Thierry/Grove-Hills, Julie/Turley, Caroline/Tompkins, Charlotte/Ciulla, Stefano/Milazzo, Vanessa/Schimmenti, Adriano/Craparo, Giuseppe (2012): European Online Grooming Project Final Report. URL: researchgate.net/publication/257941820, (03.05.2019)

Weißer Ring e.V. (2019): Tipps gegen Cyber-Grooming. URL: weisser-ring.de/praevention/tipps/cybergrooming (02.05.2019)

\section{Informationen zur Autorin}

Jennifer Vogel ist Studentin des Masterstudiengangs Lehramt für Grundschulen an der Pädagogischen Hochschule in Ludwigsburg. Im Rahmen ihrer Bachelorarbeit forschte sie zu Präventionskonzepten für Grundschüler*innen zum Schutz vor Cyber-Grooming.

\footnotetext{
Zitationshinweis:

Vogel, Jennifer (2019): Cyber-Grooming. Präventionskonzepte zum Schutz von Grundschüler*innen. In: Online-Magazin Ludwigsburger Beiträge zur Medienpädagogik, Ausgabe 20/2019. URL: medienpaed-ludwigsburg.de/
} 